\title{
THE AGGLUTININS OF TYPHOID CARRIERS
}

\author{
By ADRIANUS PIJPER, M.D., D.Sc. AND C. G. CROCKER, M.Sc. \\ From the authors' private laboratory, Pretoria, South Africa
}

Close co-operation between laboratory and public health service leaves few cases of "enteric" undetected in Pretoria. In all cases a laboratory diagnosis is carried out and an effort made to trace the source of infection. For South Africa, our population (68,000 whites and 40,000 natives and coloured) is relatively stable, and continuity in methods and comparability of results are assured by the fact that for the past 16 years all typhoid laboratory investigations have been performed in the same laboratory. In this way it has, for instance, been possible to show that in Pretoria paratyphoid infections are practically non-existent-a circumstance which greatly facilitates work on carriers. In these 16 years we have dealt with more than 3000 cases of enteric, of which several hundred were diagnosed by means of blood culture. In only two instances were paratyphoid bacilli found, and in both cases the infection could be traced to sources outside the town. During the same period we have detected nearly 60 carriers, and in every one of these typhoid bacilli were found, and not a single strain of paratyphoid bacilli.

In this paper we give the experiences collected over a number of years in tracing carriers, and also some more recent observations on Vi agglutinins in carriers.

\section{INFECTION CHIEFLY SPREAD BY CARRIERS}

Although we can trace but a relatively small number of cases of enteric directly to a carrier, we regard the carrier as the chief source of infection in our midst. Large explosive outbreaks, such as one associates with water supplies, have never occurred. We do have smaller "outbreaks", sometimes more than one in a year, and these are practically always traced to a carrier in a dairy. There are very numerous small dairies, and this fortunately limits the extent of such outbreaks. Case to case infections occur, but the majority of our cases of enteric are sporadic, and do not facilitate the search for a carrier. It is not unusual to get a single case in a household, and a carrier amongst the native servants. Here, as in dairy outbreaks, one is impressed by the large number of persons who are exposed to infection and do not become ill. There must be many channels of infection, leading from chronic carriers through ambulatory cases and "silent infections" to new sporadic cases, channels which always remain hidden, and may incidentally give rise to new chronic carriers.

Improved sanitation may, in the long run, put a stop to the carrier evil, both directly, and by decreasing the production of new carriers. But that time is not yet, and it is doubtful whether such measures will ever reach the whole 
native population of the country. Cluver (1936) reckons that 2 per cent of the total native population of the Union of South Africa are carriers, i.e. at least 100,000 persons. Their migration cannot be stopped; the carriers will come to town to work. Known carriers may slip through under a changed name.

Although we seem to have been successful with a few carriers, further experience has taught us that oral vaccination cannot be relied upon to cure carriers. Gall-bladder opérations are by no means universally successful (Eichhoff, 1932; Browning el al. 1933), and could not be universally applied in view of the large numbers to be dealt with. In any case, nothing will touch urinary carriers.

Bearing all this in mind, successive Medical Officers of Health of Pretoria have for many years directed special attention to the detection of carriers. Cure was not aimed at, but merely information, supervision, and exclusion from the more dangerous occupations. Instruction has proved satisfactory with European carriers, who form about one-half of the total number. Natives used to be repatriated to their kraals, but they often returned to town, as, for example, our case No. 1. A novel method was inaugurated by Dr F. A. Donnolly, a former Medical Officer of Health. Native carriers are now taken into the employ of the municipality, given work not connected with foodstuffs, and adequately segregated. This innovation has worked well, it safeguards the town whilst it lasts, and although most of them after a year or so go back to their kraals, they have not been known to return to town, secretly or openly.

\section{OUR METHOD OF DETECTING CARRIERS}

The local Public Health Department follows up every notification by a comprehensive enquiry into origin of food supplies and life histories of inmates of house, native servants, visitors and persons recently visited. This field work, in the hands of a most efficient inspector for many years, has built up a valuable typhoid fever dossier of the town, and with the aid of a good memory and cross-indexing often a clue, and sometimes more than one clue, is found. If several enquiries lead to one and the same source, usually a dairy, attention is at once focused on that.

The next step is the performing of blood tests. Where there is a definite clue, the number of tests to be performed is small; where there is no definite clue, and usually in the case of dairies, a large number of persons may have to be tested. This blood test does not mean a Widal. The contradictory reports in current literature about the value of agglutination tests for the detection of carriers are largely due to the fact that even modern authors just perform a Widal reaction (Roesler, 1930; Havens, 1935; Browning et al. 1933), and seem to ignore that a Widal practically means $\mathrm{H}$ agglutination only. We have met numerous carriers who possessed no $\mathrm{H}$ agglutinins, and in the list of carriers given in this paper there are three manifest carriers without any $\mathrm{H}$ agglutinins. 
From an early date, Pijper \& Pullinger (1928) suggested that a complement-fixation test could be used for the detection of carriers. Later it was shown that 7 chronic carriers all gave marked and exclusive $O$ agglutination in a serum dilution of $1: 100$ with the sensitive strain Ty 901 (Pijper, 1930). Since then we have used both our complement-fixation method and $\mathrm{O}$ agglutination in our search for carriers, completely ignoring $\mathrm{H}$ agglutination. On the whole the results of the two tests run parallel, as has been shown before (Felix, 1924; Pijper, 1930). In order to make our results more comparable with ordinary modern laboratory practice, in this paper we shall deal with agglutinins only.

In our search for carriers we take notice only of persons showing marked $(++) 0$ agglutination in a serum dilution of $1: 100$ with a sensitive strain, either "H" 901 or " $O$ " 901.

The correctness of this procedure has been confirmed in two ways. In the first place we have succeeded in detecting nearly 60 carriers in the last 10 years by examining the stools and urines of such persons as gave a positive blood test. In the second place we have repeatedly brought a beginning outbreak of enteric to an end by eliminating just those who gave a positive blood test, without waiting for confirmation by cultural methods. We are referring to outbreaks in institutions, or outbreaks connected with dairies. This observation was confirmed on a large scale by Daubenton (1931) who succeeded in reducing the incidence of enteric amongst several thousands of natives on a gold mine by the simple expedient of prohibiting all natives with positive $\mathrm{O}$ agglutination from working as cooks. On gold mines this seems both a sound and an acceptable method, but for obvious reasons it cannot be extended to the ordinary population, and certainly not as a permanent measure. Even on gold mines this rather sweeping method will become more difficult of application as time goes on, through the increasing use of vaccines.

Oral vaccination, very largely used in Pretoria, produces $\mathrm{O}$ agglutinins exclusively (Pijper \& Dau, 1930). Subcutaneous inoculations may leave some $O$ agglutinins behind (for literature see Topley \& Wilson, 1936); Grasset's new endotoxoid vaccine may produce considerable quantities (see Grasset \& Lewin, 1936). Whilst the titre of these immunization $O$ agglutinins is usually low, lower than what we regard as a positive blood test, it cannot be denied that their existence makes our method of detecting carriers more complicated. They may occur in high titres, and as the same may be said of normal agglutinins and anamnestic agglutinins, the narrowing down of the field in the search for carriers by means of $\mathrm{O}$ agglutination has its limitations. Cultural examination of our positive reactors is bound to give many negative results. Thus during the season 1934-5 our blood examinations for the purpose of detecting carriers numbered 95 , of which 13 were positive. On these 13 persons 48 stool and urine examinations were performed, and in 6 persons the carrier state was definitely established. For the season 1935-6 these figures were: total blood tests 72 , positive 25 , stools and urines examined 36 , no typhoid bacilli found. 
Apart from intermittency in the carrying, these negative cultural results may easily be due to the small number of specimens examined. Later figures are again more satisfactory.

The present position is then that by concentrating our cultural efforts on persons giving strong $O$ agglutination in a dilution 1 in 100 we have been able to find many carriers. The contention that in doing this we have missed carriers is difficult to meet, but so far we do not know of any evidence which goes against our assumption that all carriers have $O$ agglutinins. The success which has usually followed our methods of preventing typhoid fever, based on this assumption, is an argument in our favour. The only drawback of the method is that there is a large, and increasing, number of persons who possess $\mathrm{O}$ agglutinins without being carriers. This has the effect of making the method more laborious, but its application is still well within the reach of most laboratories.

\section{Vi AGGLUTININS AND CARRIERS}

Felix et al. (1935) found Vi agglutinins in significant titre $(1: 20)$ in the blood of a female carrier. This finding made us examine our available carriers for $\mathrm{Vi}$ agglutinins. Before giving our results, we wish to point out that $\mathrm{Vi}$ agglutinins are by no means so common as $\mathrm{O}$ agglutinins, neither in the normal population as in typhoid patients or convalescents. Felix et al. (1935) found appreciable quantities of Vi agglutinins in 8 out of a 100 typhoid cases, and in 5 out of 17 convalescents. Gundel \& Abdoosh (1936) had 1 positive of very low titre (1:10) amongst 21 typhoid sera, and 1 good positive amongst 11 convalescents. In 100 normal sera Horgan (1936) found traces in a serum dilution of $1: 10$ six times only, and in 28 typhoid patients, by means of repeated examinations, $\mathrm{Vi}$ agglutinin was found in a small number only. Our own observations on Vi agglutinins in patients and convalescents are rather similar to those quoted. We have also tested the sera of 70 normal persons, most of whom were natives, of the same class where carriers are often found, for the presence of $\mathrm{Vi}$ agglutinins, and have not found any. Some of these persons possessed $\mathrm{O}$ agglutinins.

Our technique for demonstrating $\mathrm{Vi}$ agglutinins is as follows: the serum is diluted 10 times, and an equal quantity of a thick suspension of typhoid bacilli, strain " $\mathrm{H}$ " 901 , killed at $56^{\circ} \mathrm{C}$, , is added. The mixture is incubated for 2 hours, and centrifuged. The clear supernatant fluid, which now is a serum dilution of $1: 20$, is put up in two rows in 1 c.c. quantities in dilutions of $1: 20$, $1: 40,1: 80$, and $1: 160$. One row receives drops of a live saline suspension of Felix's strain Ty 2, the other row similar drops of "Majora", a locally isolated "non-agglutinable" strain. Readings are taken after 2 hours in incubator and 18 hours on bench. A control tube contains the serum dilution $1: 20$, with a drop of live saline suspension of Ty " $\mathrm{H}$ " 901. "Majora" was found somewhat more agglutinable than Ty 2. 


\section{Findings in ACtual, Late ANd CURed(?) CARriers}

In the following, "Blood test pos." means a definitely positive $\mathrm{O}$ agglutination $(++)$ in a serum dilution of $1: 100$ with a live saline suspension of " $O$ " 901 , " $\mathrm{H}$ " means an agglutination of formalized suspension of $\mathrm{Ty}$ " $\mathrm{H}$ " 901, "Vi" means agglutination of a $\mathrm{Vi}$ strain after absorption as described above. For both $\mathrm{H}$ and $\mathrm{Vi}$ agglutination the titre is given.

"Stool ex." and "Urine ex." mean the planting out on suitable media in Petri dishes of the usual quantities of stool and urine within a few hours of being passed, and the subsequent picking off of suspicious colonies for further investigation, including agglutination tests. As suitable media we have used MacConkey's lactose agar and Wilson \& Blair's bismuth sulphite medium.

As all carriers upon detection are given oral vaccine made of Ty 901 , the first blood test is naturally more significant than the later ones, but the period of time between vaccination and later tests is usually large enough to make immunization agglutinins disappear to a large extent.

(1) Adult native male, was "very ill" in 1930, and in following years caused 18 cases in three different dairy outbreaks. Blood ex. pos. 19/7/32; stool ex. neg. 22/7/32; urine ex. neg. 22/7/32; blood ex. pos. 4/12/32; stool ex. neg. 4/12/32; urine ex. neg. 4/12/32; blood ex. pos. 23/10/33; stool ex. pos. 22/10/33; urine ex. neg. 22/10/33; blood ex. pos. 26/4/35; stool ex. neg. 21/7/36; stool ex. neg. 4/9/36; stool. ex. pos. 7/9/36; stool ex. neg. 8/9/36; stool ex. neg. 11/9/36; stool ex. pos. 14/9/36; stool ex. pos. 16/9/36; H pos. 13/6/36 in $1: 800$; Vi pos. $13 / 6 / 36$ in $1: 80$; Vi pos. $21 / 10 / 36$ in $1: 120$.

Comment: chronic stool carrier, possessing Vi agglutinins.

(2) Adult native male, no history of illness, but has caused at least 2 cases. Blood ex. pos. 27/12/33; stool ex. neg. 27/3/34; urine ex. neg. 27/3/34; blood ex. pos. 18/8/34; stool ex. neg. 3/9/34; urine ex. pos. 3/9/34; blood ex. pos. 12/6/36; urine ex. pos. 9/7/36; H pos. $12 / 6 / 36$ in $1: 800$; Vi pos. $12 / 6 / 36$ in $1: 200$.

Comment: chronic urinary carrier, possessing Vi agglutinins.

(3) Adult European male, had typhoid fever in 1926, has caused at least 2 cases since. Blood ex. repeatedly pos. since 1929. Urine ex. repeatedly pos., never neg., since 1929. Recently: urine ex. pos. 8/7/36; H neg. 25/6/36; Vi pos. 25/6/36 in $1: 80$.

Comment: chronic urinary carrier, possessing Vi agglutinins.

(4) Adult native male, no history of illness, closely connected with 2 cases. Blood ex. pos. 29/6/36; stool ex. neg. 6/7/36; urine ex. pos. 6/7/36; $\mathrm{H}$ neg. 6/7/36; Vi pos. 6/7/36 in $1: 120$; Vi pos. $21 / 10 / 36$ in $1: 120$.

Comment: recently discovered urinary carrier, possessing Vi agglutinins.

(5) Adult European female, had typhoid fever in 1910, had an operation for gallstones in 1915, was eventually traced as having caused at least 5 cases. Blood ex. pos. 18/11/31; stool ex. pos. 24/11/31; urine ex. neg. 24/11/31; stool ex. neg. 5/1/32; urine ex. neg. 5/1/32; stool ex. neg. 15/3/32; urine ex. neg. 15/3/32; blood ex. pos. 25/6/36; stool ex. 21/7/36 and 11 further stool ex. neg. during Sept. and Oct. 1936; H neg. 25/6/36; Vi pos. 25/6/36 in $1: 80$.

Comment: was definitely a chronic stool carrier in 1931, but recent repeated stool examinations all negative, but she has Vi agglutinins. 
(6) Adult native male, no history of illness, has at least caused 5 cases. Blood ex. pos. 4/4/34; stool ex. pos. 9/4/34; urine ex. neg. 9/4/34; blood ex. pos. 13/9/34; blood ex. pos. 19/2/36; blood ex. pos. 12/6/36; stool ex. neg. 21/7/36 and 12 further stool ex. neg. during Sept. and Oct. 1936 ; $H$ pos. $12 / 6 / 36$ in $1: 200$; Vi pos. $12 / 6 / 36$ in $1: 40$.

Comment: was definitely a stool carrier in 1934, but recent repeated stool examinations all negative, and has $\mathrm{Vi}$ agglutinins.

(7) Adult native male, no history of illness, caused 1 case. Blood ex. pos. 15/1/34; stool ex. pos. 18/1/34; urine ex. neg. 18/1/34; stool ex. neg. 26/1/34; urine ex. neg. 26/1/34; stool ex. neg. 5/2/34; urine ex. neg. 5/2/34; blood ex. pos. 24/4/34; blood ex. pos. 12/6/36; stool ex. neg. 21/7/36 and 12 further stool ex. neg. during Sept. and.Oct. 1936; H neg. 12/6/36; Vi pos. $12 / 6 / 36$ in $1: 20$.

Comment: was definitely a stool carrier in 1934, but recent repeated stool examinations all negative, and has Vi agglutinins.

(8) Adult European female, had typhoid fever at end of 1931, and then had investigations, performed to exclude possibility of becoming a carrier. Stool ex. neg. 26/11/31; urine ex. pos. 26/11/31; stool ex. neg. 5/1/32; urine ex. neg. 5/1/32; urine ex. pos. 9/3/32; urine ex. pos. 5/7/32; urine ex. neg. 30/3/33; urine ex. neg. 25/6/36 and 13 further neg. urine ex. during Aug., Sept. and Oct. 1936; blood ex. pos. 25/6/36; H neg. 25/6/36; Vi neg. 25/6/36; Vi neg. 6/8/36.

Comment: was obviously a temporary carrier for at least 8 months after having typhoid fever, seems to be no longer a carrier, and has no Vi agglutinins.

(9) Female European baby, had undiagnosed typhoid fever in Jan. 1935, infected 3 in household after recovery in Feb. 1935. Stool ex. neg. 16/2/35; urine ex. pos. 16/2/35; stool ex. neg. 21/3/35; urine ex. pos. 21/3/35; stool ex. neg. 29/10/35; urine ex. neg. 29/10/35 and 8 further urine ex. neg. during Sept. and Oct. 1936; blood ex. pos. 29/10/35; blood ex. pos. 25/6/36; H neg. 25/6/36; Vi neg. 25/6/36; Vi neg. 6/8/36.

Comment: was obviously a temporary carrier early in 1935 , seems to be no longer a carrier, and has no Vi agglutinins.

(10) Adult native male, no history of illness, has caused at least 5 cases. Blood ex. pos. 6/4/36; stool ex. pos. 11/4/34; urine ex. neg. 11/4/34; blood ex. pos. 13/2/35; blood ex. pos. 12/6/36; stool ex. neg. 21/7/36 and 12 further stool ex. neg. during Sept. and Oct. 1936; $\mathrm{H}$ neg. 12/6/36; Vi neg. 12/6/36.

Comment: was definitely a stool carrier in 1934, but recent repeated stool examinations all negative, and has no $\mathrm{Vi}$ agglutinins now.

(11) Adult female Indian, had typhoid fever in 1929, in later years caused several cases. Blood ex. pos. 20/1/33; stool ex. pos. 24/1/33; urine ex. neg. 24/1/33; blood ex. pos. 25/6/36; stool ex. neg. 25/6/36; urine ex. neg. 25/6/36; stool ex. neg. 22/7/36 and 12 further stool ex. neg. during Sept. and Oct. 1936; H neg. 25/6/36; Vi neg. 25/6/36.

Comment: was definitely a stool carrier in 1933, but recent repeated stool examinations negative, and has no $\mathrm{Vi}$ agglutinins now.

(12) Adult native male, no history of illness, has caused a dairy outbreak in 1933. Blood ex. pos. 30/11/33; stool ex. neg. 2/12/33; urine ex. pos. 2/12/33; blood ex. pos. 26/4/34; stool ex. neg. 14/12/34; urine ex. neg. 14/12/34; blood ex. pos. 13/2/35; stool ex. neg. 8/1/35; 
urine ex. neg. 8/1/35; blood ex. pos. 12/6/36; urine ex. neg. 9/7/36 and 13 further urine ex. neg. during Aug., Sept. and Oct. 1936; H neg. 12/6/36; Vi neg. 12/6/36.

Comment: was definitely a urinary carrier in 1933, but recent repeated urine examinations all negative, and has no Vi agglutinins now.

(13) Adult European male, had typhoid fever in 1908, has 6 cases traced to him between 1918 and 1932. Blood ex. pos. 13/2/32; stool ex. pos. 17/2/32; urine ex. neg. 17/2/32; stool ex. neg. 28/4/32; urine ex. neg. 28/4/32; blood ex. neg. 26/9/36; Vi neg. 26/9/36.

Comment: was definitely a stool carrier in 1932, has no $\mathrm{Vi}$ agglutinins now, no recent cases traced to him.

(14) Adult native male, no history of illness, discovered in routine examination of dairy natives, has no cases traced to him. Blood ex. pos. 6/3/28; urine ex. pos. 11/3/28; urine ex. neg. 6/4/30; stool ex. neg. 20/4/32; urine ex. pos. 20/4/32; blood ex. pos 26/4/34; urine ex. neg. 7/1/35; urine ex. neg. 8/6/36; blood ex. pos. 29/9/35; urine ex. neg. 29/9/36; Vi neg. 29/9/36.

Comment: obviously a chronic urinary carrier from 1928 till 1932; seems then to have lost his bacilli, and now has no Vi agglutinins.

\section{SUMMARY AND DISCUSSION}

By limiting our cultural investigations to persons who possess appreciable quantities of $\mathrm{O}$ agglutinins, we have detected a number of typhoid carriers and there are no indications that any carriers have been missed by following this labour-saving procedure. Several of our carriers were found to possess no $\mathrm{H}$ agglutinins.

As, however, $\mathrm{O}$ agglutinins occur in persons who are not carriers, and the number of such positive $O$ reactors is increasing through the use of vaccines, the number of cultural examinations where no positive results can be expected is growing at a rate which will gradually make our procedure more difficult of application.

It would seem that $\mathrm{Vi}$ agglutination might provide a new and efficient method of narrowing down the field for cultural examinations. Vi agglutinins are very rare in normal persons. We have 4 manifest chronic carriers who possess significant quantities of $\mathrm{Vi}$ agglutinins. We have 3 persons who at one time were chronic stool carriers, in whom we now also find significant quantities of $\mathrm{Vi}$ agglutinins, but in whom at the time we cannot demonstrate typhoid bacilli. We also have 5 persons who at one time were chronic carriers, who now do not show any $\mathrm{Vi}$ agglutinins, and in whom we also at this time cannot find typhoid bacilli. Two of these were urinary carriers, which makes their negative cultural results more significant. Lastly, we have 2 persons who at one time obviously were temporary urinary carriers, and who now have lost their bacilli, and in whom also we now cannot detect Vi agglutinins.

In the search for typhoid carriers negative cultural results carry little weight. Our positive findings support the view that typhoid carriers are characterized by the possession of significant quantities of $\mathrm{Vi}$ agglutinins. 
Acknowledgements. We wish to thank Drs J. J. Boyd, G. D. Laing, and F. A. Donnolly, late Medical Officers of Health of Pretoria, for their support in these investigations. Our special thanks are due to the present Medical Officer of Health, Dr H. Nelson, for his generous co-operation.

\section{REFERENCES}

Browning, C. H. et al. (1933). Spec. Rep. Ser., Med. Res. Counc. London, No. 179.

Cluver, E. H. (1936). Quart. Bull. Health Organ. League of Nat. 5, 1. Geneva.

Daubenton, F. (1931). J. Med. Assoc. S. Africa, 5, 71.

Eтснноғт, E. (1932). Die Chirurgie der chronischen Typhus- und Paratyphusbazillenausscheider. Jena.

Felix, A. (1924). J. Immunol. 9, 115.

Felix, A., Krikorian, K. S. \& Reitler R. (1935). J. Hygiene, 35, 421.

Grasset, E. \& Lewin, W. (1936). Brit. J. exp. Path. 17, 179.

Gundel, M. \& Aвdoosh, Y. B. (1936). Zbl. Bakter. I Orig. 136, 54.

Havens, L. C. (1935). The Bacteriology of Typhoid, Salmonella, and Dysentery Infections and Carrier States. New York.

Horgan, E. S. (1936). J. Hygiene, 36, 368.

Pijper, A. (1930). Ibid. 29, 380.

PiJper, A. \& DaU, H. (1930). Brit. J. exp. Path. 11, 112.

Pijper, A. \& Pullinger, B. D. (1928). Brit. Med. J. 1, 2.

RoesLer, G. (1930). Das Schrifttum zur Frage der Typhus-und Paratyphusbazillenausscheider. Jena.

Topley, W. W. C. \& Wirson, G. S. (1936). The Principles of Bacteriology and Immunity. London.

(MS. received for publication 21. xII. 1936.-Ed.) 